\title{
Effect of neoadjuvant treatment with anastrozole on tumour histology in postmenopausal women with large operable breast
}

\section{cancer}

\author{
TJ Anderson', JM Dixon², M Stuart ${ }^{3}$, T Sahmoud $^{3}$ and WR Miller*,2 \\ 'Department of Pathology, Westem General Hospital, Edinburgh EH4 2XU, UK; ${ }^{2}$ Breast Unit, Western General Hospital, Edinburgh EH4 2XU, UK; \\ ${ }^{3}$ AstraZeneca, Alderley Park, Cheshire SKIO 4TG, UK
}

Anastrozole is an orally active, non-steroidal aromatase inhibitor which appears effective as neoadjuvant treatment of breast cancer. Histological changes have been evaluated in biopsies from large, oestrogen-receptor rich, operable breast tumours in postmenopausal women following 12 weeks of neoadjuvant anastrozole treatment $(\mid \mathrm{mg}(n=\mid 2)$ or $\mid 0 \mathrm{mg}(n=1 \mid))$. Of the 23 patients, 18 had a clinical response following treatment. Compared with pre-treatment biopsies anastrozole-treated specimens displayed decreased cellularity and/or increased fibrosis in 15 tumours; changes in gland formation, nuclear pleomorphism, or mitoses, in 12 cases; and a reduction in Mib I score in all tumours. Marked changes in apoptotic scores were seen following treatment but the direction of effect was inconsistent. In all 17 tumours which were positive for progesterone receptors before therapy, treatment was associated with reduced staining for progesterone receptors. There was no consistent effect of treatment on oestrogen-receptor expression. It is concluded that neoadjuvant anastrozole treatment in this patient group has marked effects on tumour histopathology but these do not always correlate with clinical response. British Journal of Cancer (2002) 87, 334-338. doi:|0.1038/sj.bjc.6600435 www.bjcancer.com

(C) 2002 Cancer Research UK

Keywords: neoadjuvant; anastrozole; postmenopausal women; histology; pathology

\begin{abstract}
Although surgery is the most important mode of primary treatment for most breast cancers, neoadjuvant treatment can be a useful part of the clinical management of many patients. By giving appropriate drugs before surgery, tumours may shrink such that less extensive surgery is needed and, in particular, allow breastconserving surgery in patients who might otherwise have required a mastectomy before neoadjuvant treatment (Miller et al, 1999). Neoadjuvant treatment also provides a valuable opportunity to study the effect of drugs on a variety of biochemical and histological features of the tumour. Whilst chemotherapy is the most frequent therapy used in the neoadjuvant setting, endocrine therapy has also been evaluated in hormone-sensitive, large, operable or locally advanced breast cancer (Anderson et al, 1989; Valero et al, 1989; Leal da Silva et al, 1998).

Anastrozole is a potent, non-steroidal inhibitor of aromatase, the enzyme responsible for catalysing the conversion of androgens to oestrogen. The drug markedly suppresses oestrogen levels in postmenopausal women, in whom peripheral aromatase activity is the main route of oestrogen production (Miller, 1996; Bajetta et al, 1999). Clinical trials in postmenopausal women with advanced breast cancer have shown that anastrozole is well tolerated (Buzdar et al, 1998). When used as second-line therapy in patients who have progressed on tamoxifen treatment, it is significantly superior to megestrol acetate in terms of overall survival (Buzdar et al, 1998). In addition, anastrozole has greater efficacy in terms of prolonged time to progression, compared with tamoxifen, as
\end{abstract}

*Correspondence: Professor WR Miller; E-mail: w.r.miller@ed.ac.uk Received 20 December 200 I; revised 22 April 2002; accepted 8 May 2002 first-line therapy for patients with advanced breast cancer known to be hormone receptor-positive (Buzdar et al, 2000; Nabholtz et al, 2000; Bonneterre et al, 2001), as well as having tolerability advantages. Thus, anastrozole is now challenging the place of tamoxifen as first-line treatment of advanced breast cancer. Studies have indicated that anastrozole is also effective in the neoadjuvant setting, producing clinically important reductions in tumour volume and mastectomy rates (Dixon et al, 2000).

Although it has been established that anastrozole suppresses oestrogen levels within the tumour when used for neoadjuvant treatment (Geisler et al, 1999), the precise mechanisms by which this oestrogen deprivation exerts its clinical effect are not well understood. The aims of this study were therefore to determine the effects of 12 weeks' treatment with anastrozole on the morphology, histological grade, proliferative activity, and steroid receptor status of breast cancers, in order to gain a greater understanding of the mode of action of this drug.

\section{MATERIALS AND METHODS}

The study was approved by the appropriate ethics committee, and was conducted in accordance with the ethical principles of the Declaration of Helsinki. All patients gave written informed consent before any trial-related procedures were performed.

\section{Patients}

The study included postmenopausal women with large, operable, oestrogen-receptor (ER)-rich (ER shown on the initial core biopsy to be $>80$ by histoscore) breast cancer. Postmenopausal status was 
defined as patients aged 50 years or over who had not menstruated in the past 12 months, or women of any age with follicle-stimulating hormone (FSH) levels $>40 \mathrm{IU} \mathrm{l}^{-1}$. For inclusion in the study, patients had to have operable breast cancers $>3 \mathrm{~cm}\left(\mathrm{~T}_{2}>3 \mathrm{~cm}\right.$, $\mathrm{T}_{3}$ ) or locally advanced breast cancer $\left(\mathrm{T}_{4 \mathrm{~b}}\right)$. Full demographic and baseline tumour characteristics of the patients have been reported previously (Dixon et al, 2000) and are summarized in Table 1.

\section{Study design}

The results presented here are secondary outcomes from a previously published single-centre study which was designed primarily to assess the effects of anastrozole on peripheral and intra-tumour aromatase activity (Dixon et al, 2000). Patients were randomized in a $1: 1$ ratio to double-blind neoadjuvant treatment with anastrozole 1 or $10 \mathrm{mg}$ (once daily) for 12 weeks. Before anastrozole treatment started, breast tissue was removed by wedge biopsy to determine variables including baseline morphology, histological grade, proliferative activity, and steroidal receptor status. At the end of the 12-week treatment period, tumours were removed by curative-intent surgery. The tumours removed during surgery were analysed to determine the effects of treatment on the above variables.

\section{Assessments}

Tumour volume Our previous experience, based on excision biopsies, has shown that ultrasound measurements are generally more accurate than calliper or mammographic assessments (Forouhi et al, 1994). Therefore, tumour volume was assessed pretreatment and at monthly intervals by ultrasound, as previously described (Dixon et al, 2000). Results were calculated as the percentage change in volume between measurements made before treatment and after three months' therapy. In previous publications (Bajetta et al, 2000; Zilembo et al, 2000) a reduction of $>25 \%$ in tumour volume is taken as evidence of clinical response (assessments made in accordance with the International Union Against Cancer guidelines). However, in the present study, all tumour volume reductions were greater than $50 \%$.

Histopathology The histopathological features of the tumour removed by surgery at the end of the study were compared with the initial wedge biopsy. Tumour morphology was judged by comparing changes in cellularity and fibrosis. Histology was also scored for grading features according to the method described by Elston and Ellis (1991). This method involves the histological assessment of three components of tumour morphology: tubule formation, nuclear pleomorphism and frequency of mitoses, and an overall tumour grade ( 1 to 3 ) is calculated.

Table I Demographic and baseline tumour characteristics of patients

\begin{tabular}{lcc}
\hline Characteristics & $\begin{array}{c}\text { Anastrozole } \\
\text { I mg day } \\
(\mathbf{n = 1}\end{array}$ & $\begin{array}{c}\text { Anastrozole } \\
\mathbf{I 0} \mathbf{~ m g ~ d a y ~}^{-\mathbf{I}} \\
(\mathbf{n = 1}\end{array}$ \\
\hline Age (years) & $74.1 \pm 8.7$ & $69.6 \pm 8.8$ \\
Weight $(\mathrm{kg})$ & $69.6 \pm 9.6$ & $75.7 \pm 13.4$ \\
Height $(\mathrm{cm})$ & $156.3 \pm 12.2$ & $158.1 \pm 12.2$ \\
Tumour stage* & $11(91.7)$ & $9(81.8)$ \\
$\mathrm{T}_{2}$ & $0(0)$ & $2(18.2)$ \\
$\mathrm{T}_{3}$ & $1(8.3)$ & $0(0)$ \\
$\mathrm{T}_{4 \mathrm{~b}}$ & $2(16.7)$ & $1(9.1)$ \\
Lymph node metastases & & \\
\hline
\end{tabular}

All values are mean \pm s.d., except: * number (\%).
Cell proliferation - Mib1 staining Ki67 antigen was assayed, by measuring the binding of a mouse monoclonal antibody, Mib1, to the Ki67 nuclear antigen, using sections taken from a pre-treatment tumour biopsy sample and from a post-treatment surgical specimen. The percentage of cells staining in a minimum of 10 representative high-power fields was used to quantify Mib1 expression (Gerdes et al, 1983).

Apoptotic index Apoptotic (cell death) index was measured using the TUNEL immunohistochemical technique (Lebat-Moleur et al, 1998). The index was defined as the number of apoptoses per 1000 cells and was derived using methodology originally used to assess mitotic index (Simpson et al, 1992).

Oestrogen and progesterone receptors Oestrogen receptor and progesterone receptor (PgR) status were assessed by immunohistochemical techniques after microwave antigen retrieval, using ID5 (Dako) for ER status and PG88 (Biogenix) for PgR status. The results were scored on a scale of 0 to 3 for intensity (with each successive score denoting increasing intensity), and on a score of 0 to 5 for proportion (with the greatest proportion denoted by a score of 5); the values were then summed into a category score with a range of 0 to 8 (Allred et al, 1998).

\section{Statistical methods}

The study size was chosen in order to analyse the primary endocrinological endpoints. The statistic analyses for pathology parameters were therefore applied without previous power calculations. Because numbers of patients in each dose group were small and there is no a priori reason to believe that pathology responses are dose-related, analyses have been performed combining the dose groups (although for information tables and figures present data separately).

\section{RESULTS}

A total of 12 patients were eligible for analysis in the anastrozole $1 \mathrm{mg}$ group, and a total of 11 patients were available in the anastrozole $10 \mathrm{mg}$ group.

\section{Histopathological assessments}

Marked morphological changes with treatment were evident in the majority of tumours (15 of $23 ; 65 \%$ ). These constituted both decreased cellularity and increased fibrosis in 11 cases, and decreased cellularity alone in two; in two tumours there were only microscopic foci of disease after treatment. All these effects were taken as evidence of a pathology response. Additionally, changes in grading characteristics were associated with treatment in 12 of the tumour pairs. Tubular features were increased in five cases, nuclear pleomorphism was decreased in four, and mitotic index was decreased in five cases but increased in one.

The relationship between pathological response and ultrasound volume change is shown in Table 2. This indicates that pathological changes with treatment may be observed in tumours that have no

Table 2 The relationship between clinical and pathological response

\begin{tabular}{lcc}
\hline & \multicolumn{2}{c}{ Clinical response } \\
\cline { 2 - 3 } & $<\mathbf{5 0 \%}$ & $>\mathbf{5 0 \%}$ \\
\hline Pathological response & 2 & 13 \\
No change & 3 & 5 \\
Total & 5 & 18 \\
\hline
\end{tabular}


ultrasound response. Conversely, whilst the majority of tumours with a major reduction in ultrasound volume also show pathological response, five tumours (of 18) were without evidence of pathological changes. The median tumour volume reduction in tumours with a pathological response was $78.3 \%$ (range $=16.5$ $97.5 \%$ ) and for the no-change category was $67.5 \%$ (range $=6.4-$ $94.4 \%)$, the difference between the groups was non-significant $(P=0.59$ by Wilcoxon rank test).

\section{Marker immunohistochemistry}

Mib1 staining Measurements of Mibl expression showed that treatment with anastrozole was associated with a reduction in staining score in all cases (Figure 1). The initial levels and degree of reduction was not significantly different in tumours with and without pathological response.

TUNEL Changes in apoptotic index were noted with treatment (Figure 2). However, the direction of change was not consistent, decreasing in 12 tumours and increasing in 11 tumours. Furthermore, pattern of change was not related to pathological response.
Oestrogen and progesterone receptors In terms of effects on ER expression, treatment was associated with no change in the intensity and proportion of cells staining in 15 cases; in the remaining eight tumours there were minor changes in category score for staining parameters, but these were minor and equally increased or decreased.

Of the 23 tumours, 18 were assessed as being PgR positive before treatment (Table 3), scoring between category 3 and 8 ; of these, $11(61 \%)$ subsequently displayed a pathological response. Of the five PgR-negative tumours, four (80\%) showed evidence of pathological response and none changed their PgR status. However, anastrozole treatment caused a marked reduction in expression of $\mathrm{PgR}$ in 17 of the 18 receptor-positive tumours (in 11 cases this was a total loss). This reduction in expression was irrespective of pathological response.

\section{DISCUSSION}

The results of this study show that neoadjuvant treatment with anastrozole may have marked effects on histopathological features within breast tumours. These include changes in histological grading features, markers of proliferation and cell death, and of PgR

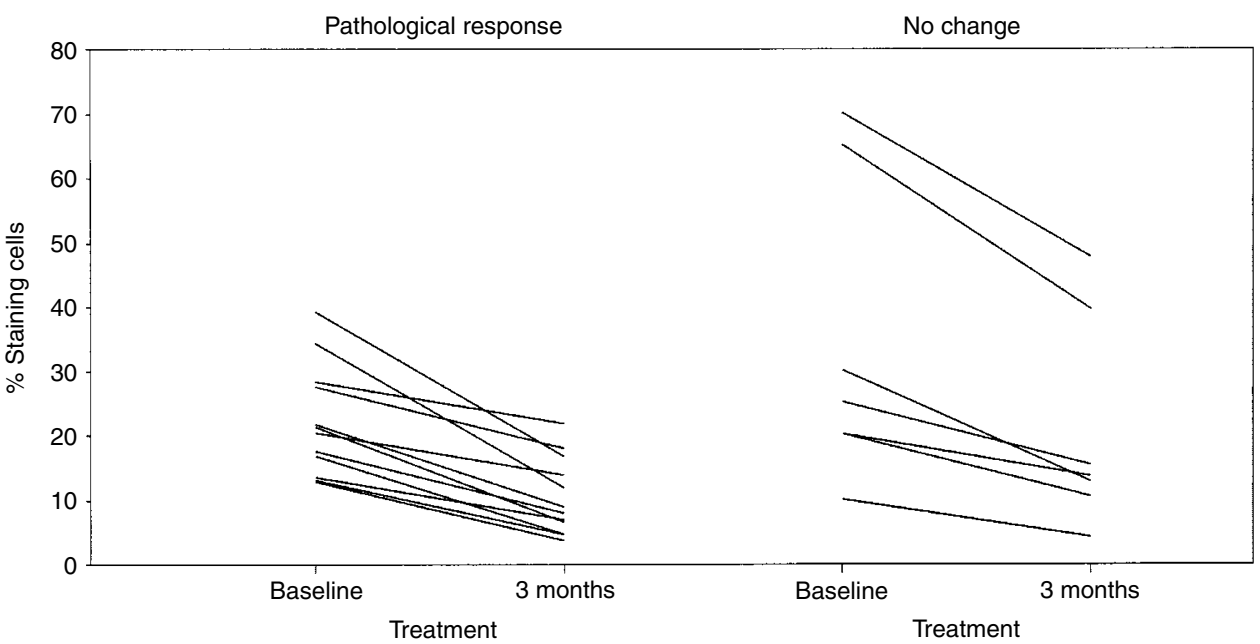

Figure I Changes in Mib I (\%) after 12 weeks of treatment with anastrozole (combination of I and I0 mg doses) in tumours with a pathological response to treatment (left panel) and in tumours with no response to treatment (right panel).

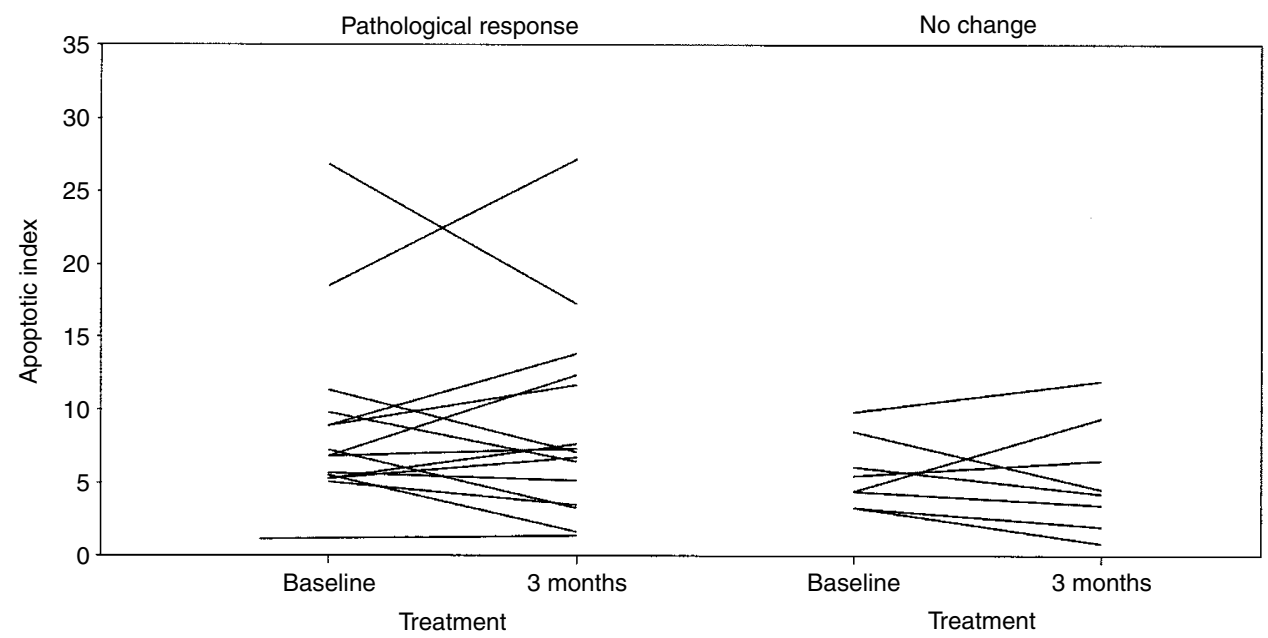

Figure 2 Changes in apoptotic index (\%) after 12 weeks of treatment with anastrozole (combination of I and 10 mg doses) in tumours with a pathological response to treatment (left panel) and in tumours with no response to treatment (right panel). 
Table 3 The relationship between change in progesterone receptors and pathological response

\begin{tabular}{lcccc}
\hline & \multicolumn{2}{c}{ Receptor positive } & Receptor negative \\
& Lost & Decreased & No change & change \\
\hline $\begin{array}{l}\text { Pathological } \\
\text { repsonse }\end{array}$ & 6 & 5 & 0 & 4 \\
No change & 5 & 1 & 1 & 1 \\
Total & 11 & 6 & 1 & 5 \\
\hline
\end{tabular}

expression. We believe that this range of observation has never been previously reported for an aromatase inhibitor. It is therefore important to discuss these findings in terms of: (1) their relationship to clinical response, (2) their inter-relationships, and (3) a comparison with the effects of other endocrine agents, most notably tamoxifen.

Clear pathological responses in terms of reduced cellularity and/or increased fibrosis were noted in the majority of tumours (15 of $23 ; 65 \%)$. This high response rate probably reflects the patient selection criterion based on ER-rich tumours. It is, however, lower than the clinical response frequency in the same tumours. Furthermore, pathological response only correlated poorly with clinical response as determined by ultrasound. Thus, although the majority of tumours that had a substantial volume reduction with treatment also showed a pathological response (13 of 18), a minority had an ultrasound response without evidence of pathological changes. This is perhaps not surprising since it is possible to visualize a scenario in which tumours shrink without altering their microscopic morphology. Conversely, most tumours failing to show a significant reduction in tumour volume were also without evidence of pathological change. Furthermore, there were two tumours that did not have a significant reduction in tumour volume by ultrasound but yet had a pathological response. In these particular cases it may be that ultrasound measurements (which in our hands generally appear to give the most accurate assessment of tumour burden (Forouhi et al, 1994) did not accurately reflect true response. Indeed, calliper assessments in these particular cases indicated reductions in tumour size (54 and 99\%) which were more compatible with the small pathological size of the excised lesion (data not shown). These observations suggest that, in monitoring the response to endocrine agents, pathological determinants should ideally supplement more traditional clinical tumour measurements.

In addition to changes in cellularity, other pathological features were altered with treatment in approximately $50 \%$ of cases: these included tubule formation, decreased nuclear pleomorphism, and decreased mitosis, which occurred both alone or in combination. This indicates that anastrozole therapy is capable of modulating cellular populations within individual tumours. Furthermore, these changes are generally towards a phenotype that is accepted as being less aggressive.

The effects of treatment with anastrozole on Mib1 expression were striking and consistent in that the proportion of staining cells was always less in the treated tumour as compared with the pre-treatment biopsy. Whilst the degree of reduction varied widely between different tumour pairs, this was not related to pathological changes or degree of tumour shrinkage, ER level or PgR status. It would therefore seem that one of the more important mechanisms of action of anastrozole, at least in its chronic use, is to take tumour cells out of the cycle of division. However, whether this then translates into a clinical or pathological response depends on other factors. The practical implication of these observations is that measurement of Mibl staining, either initially or after 3 months' treatment, is not helpful in predicting/monitoring response. Whether intermediate time-point measurements would have had more utility is unresolved, but is worthy of further study. It is interesting to note that whilst we and others have observed consistent decreases in proliferation following therapy with aromatase inhibitors (Geisler et al, 2001; Harper-Wynne et al, 2002), this was not our experience with tamoxifen (Keen et al, 1997). Tamoxifen treatment could be associated with an increase in proliferation markers, particularly in non-responding tumours. Effects on such markers may be representative of an important difference in the mechanism of action between tamoxifen and aromatase inhibitors.

Changes in apoptotic index were observed with treatment. However, the direction of change was not consistent and did not relate to clinical or pathological response or change in Mib1 score. Assessment of these changes in apoptosis is complicated by the event being: (1) transient and of low frequency in most breast cancers, and (2) the result of either a primary response to treatment (successful therapy would increase score) or being secondary to changes in proliferation (decreased proliferation would produce a fall in score). Given that the chronology of the processes of proliferation and apoptosis may differ in individual tumours, the assessment of apoptosis at a single time-point of 3 months into treatment is almost certainly suboptimal. Given that, in experimental models, the primary apoptotic response to endocrine therapy precedes that of proliferation and may be seen as early as $2-3$ days (Cameron et al, 2000), future studies of neoadjuvant anastrozole would benefit from the inclusion of tumour samples early in the treatment protocol. These studies are currently underway.

The lack of a consistent effect on ER expression observed in this study is also in agreement with results reported previously with other aromatase inhibitors (Sasano et al, 1999; Harper-Wynne et $a l, 2002)$. This contrasts with influences on the PgR, the expression of which was reduced in all but one case and was totally lost with treatment in most cases. The results of the present study are similar to previously reported data on other aromatase inhibitors (Sasano et al, 1999; Harper-Wynne et al, 2002) and are compatible with the PgR being an oestrogen-inducible protein. It should be noted that the decrease in PgR occurred in both tumours, with and without clinical or pathological response. Lack of clinical or pathological response is therefore not because the tumour fails to recognize anastrozole as an oestrogen-depriving therapy. It should be noted that in this setting, PgR status in the pre-treatment biopsy was not predictive of clinical or pathological responses: for example, four of the five PgR-negative tumours exhibited clear pathological responses with treatment. The consistent effect of anastrozole in decreasing PgR staining is further evidence that the drug's mode of action differs from that of tamoxifen, which has variable effects on PgR, including increased expression (Miller, 1996; Miller et al, 1999; Chang et al, 2000). The difference in phenotypic expression following treatment with tamoxifen and aromatase inhibitors may have clinical relevance in terms of resistance to treatment and the choice of subsequent sequence of therapies ( $\mathrm{Hu}$ et al, 1993; MacGregor and Jordan, 1998). Interestingly, aromatase inhibitors have already been shown to be of therapeutic value for many patients whose tumours have developed resistance to tamoxifen (Bajetta et al, 1999).

In summary, the neoadjuvant use of anastrozole has been shown to have clear effects on histological features of breast tumours. The pathological findings that anastrozole decreases the expression of both $\mathrm{PgR}$ and Mibl are particularly striking, and reflect the powerful anti-oestrogenic and anti-proliferative potential of the drug. However, the present study should be regarded as a pilot study, and the intermediary mechanisms by which anastrozole achieves a clinical or pathological response remain unclear. Further research in this area should include a larger study population, and samples taken earlier after the start of treatment. 


\section{REFERENCES}

Allred DC, Harvey JM, Berardo M, Clark GM (1998) Prognostic and predictive factors in breast cancer by immunohistochemical analysis. Mod Patho 11: $155-168$

Anderson ED, Forrest AP, Levack PA, Chetty U, Hawkins RA (1989) Response to endocrine manipulation and oestrogen receptor concentration in large operable primary breast cancer. Br J Cancer 60: 223-226

Bajetta E, Zilembo N, Bichisao E (1999) Aromatase inhibitors in the treatment of postmenopausal breast cancer. Drugs Aging 15: $271-283$

Bajetta E, Zilembo N, Bichisao E, Martinetti A, Buzzoni R, Pozzi P, Bidoli P, Ferrari L, Celio L (2000) Tumor response and estrogen suppression in breast cancer patients treated with aromatase inhibitors. Ann Oncol 11: $1017-1022$

Bonneterre J, Buzdar A, Nabholtz JM, Robertson JFR, Thurlimann B, von Euler M, Sahmoud T, Webster A, Steinberg M (2001) Anastrozole (Arimi$\mathrm{dex}$ ) is superior to tamoxifen as first-line therapy in hormone receptorpositive advanced breast cancer: results of two randomized trials designed for combined analysis. Cancer 92: 2248-2258

Buzdar AU, Jonat W, Howell A, Jones SE, Blomqvist CP, Vogel CL, Eiermann W, Wolter JM, Steinberg M, Webster A, Lee D, for the Arimidex Study Group (1998) Anastrozole versus megestrol acetate in the treatment of postmenopausal women with advanced breast carcinoma. Results of a survival update based on a combined analysis of data from two mature Phase III trials. Cancer 83: $1142-1152$

Buzdar A, Nabholtz JM, Robertson JF, Thurlimann B, Bonneterre J, von Euler M, Steinberg M, Webster A. (2000) Anastrozole (Arimidex) versus tamoxifen as first-line therapy for advanced breast cancer in postmenopausal women. Combined analysis from two identically designed multicenter trials. Proc ASCO, 154a: Abstract 609D

Cameron DA, Keen JC, Dixon JM, Bellamy C, Hanby A, Anderson TJ, Miller WR (2000) Effective tamoxifen therapy of breast cancer involves both antiproliferative and pro-apoptotic changes. Eur J Cancer 36: 845 - 851

Chang J, Powles TJ, Allred DC, Ashley SE, Makris A, Gregory RK, Osborne CK, Dowsett M (2000) Prediction of clinical outcome from primary tamoxifen by expression of biologic markers in breast cancer patients. Clin Cancer Res 6: 616-621

Dixon JM, Renshaw L, Bellamy C, Stuart M, Hoctin-Boes G, Miller WR (2000) The effects of neoadjuvant anastrozole (Arimidex) on tumour volume in postmenopausal women with breast cancer: a randomized, double-blind, single-center study. Clin Cancer Res 6: 2229-2235

Elston CW, Ellis IO (1991) Pathological prognostic factors in breast cancer. I. The value of histological grade in breast cancer: experience from a large study with long-term follow-up. Histopathology 19: 403-410

Forouhi P, Walsh JS, Anderson TJ, Chetty U (1994) Ultrasonography as a method of measuring breast tumour size and monitoring response to primary systemic treatment. Br J Surg 81: $223-225$

Geisler J, Bernsten H, Ottestad L, Lindtjorn B, Dowsett M, Lonning PE (1999) Neoadjuvant treatment with anastrozole ('Arimidex') causes profound suppression of intra-tumor estrogen levels. Proc ASCO 18: Abstract No 311

Geisler J, Detre S, Berntsen H, Ottestad L, Lindtjorn B, Dowsett M, Lonning PE (2001) Influence of neoadjuvant anastrozole (Arimidex) on intratumoral estrogen levels and proliferation markers in patients with locally advanced breast cancer. Clin Cancer Res 7: 1230-1236
Gerdes J, Schwab U, Lembe H, Stein H (1983) Production of a mouse monoclonal antibody reactive with a human nuclear antigen associated with cell proliferation. Int J Cancer 31: 13-20

Harper-Wynne CL, Sacks NPM, Shenton K, MacNeill FA, Sauven P, Laidlaw IJ, Rayter Z, Miall S, Howes A, Salter J, Hills MJ, Lowe FM, A'Hern R, Nasiri N, Doody D, Iqbal J, Dowsett M (2002) Comparison of the systemic and intratumoral effects of tamoxifen and the aromatase inhibitor vorozole in postmenopausal patients with primary breast cancer. J Clin Oncol 20: $1026-1035$

Hu XF, Veroni M, De Luise M, Wakeling, Sutherland R, Watts CK (1993) Circumvention of tamoxifen resistance by the pure anti-estrogen ICI 182,780. Int J Cancer 55: $873-876$

Keen JC, Dixon JM, Miller EP, Cameron DA, Chetty U, Hanby A, Bellamy C, Miller WR (1997) The expression of Ki-S1 and BCL-2 and the response to primary tamoxifen therapy in elderly patients with breast cancer. Breast Cancer Res Treat 44: 123-133

Leal da Silva JM, Cardosa F, Oliveira F, Cunha H, Pinto Ferreira E (1998) Neoadjuvant hormonal therapy in locally advanced breast cancer. Eur J Cancer 34: S15 Abs 52

Lebat-Moleur F, Guillermet C, Lorimier P, Robert C, Lantuejoul S, Brambilla E, Negoescu A (1998) TUNEL apoptotic cell detection in tissue selections: critical evaluation and improvement. J Histochem Cytochem 46: 327 - 334

MacGregor JI, Jordan VC (1998) Basic guide to the mechanisms of antiestrogen action. Pharmacol Rev 50: 151-196

Miller WR (1996) Aromatase inhibitors. Endocrine-Related cancer 3: 65-79

Miller WR, Anderson TJ, Hawkins RA, Keen J, Dixon JM (1999) Neoadjuvant endocrine treatment: the Edinburgh experience. ESO Scientific Updates 4: 89-99

Miller WR, Stuart M, Sahmoud T, Dixon JM. Anastrozole ('Arimidex') blocks oestrogen synthesis both peripherally and within the breast in postmenopausal women with large operable breast cancer. $\mathrm{Br} J$ Cancer (submitted)

Nabholtz JM, Buzdar A, Pollak M, Harwin W, Burton G, Mangalik A, Steinberg M, Webster A, von Euler M (2000) Anastrozole is superior to tamoxifen as first-line therapy for advanced breast cancer in postmenopausal women - results of a North American multicenter randomized trial. $J$ Clin Oncol 18: $3758-3776$

Sasano H, Sato S, Ito K, Yajima A, Nakamura J, Yoshihama M, Ariga K, Anderson TJ, Miller WR (1999) Effects of aromatase inhibitors on the pathobiology of human breast, endometrial and ovarian carcinoma. Endocrine-Related cancer 6: $197-204$

Simpson JF, Dutt PL, Page DL (1992) Expression of mitoses per thousand cells and cell density in breast carcinomas: a proposal. Hum Pathol 23: $608-611$

Valero V, Hoff PM, Singletary SE, Buzdar AU, Theriault RL, Strom E, Booser DJ, Asmar L, Frye D, McNeese MD, Hortobagyi GN (1989) Combined modality treatment of locally advanced breast cancer (LABC) in elderly patients (PTS) using tamoxifen (TAM) as primary therapy. Proc Am Soc Clin Oncol 17: 105a Abstract 403

Zilembo N, Bajetta E, Bichisao E, Martinetti A, Pozzi P, Ferrari L, La Torre I, Celio L (2000) Estrogen suppression (ES) and tumor response in breast cancer (BC) patients treated with aromatase inhibitors. Proc of ASCO Abstract 399 